\title{
Abrupt Aggravation of Encapsulated Seroma after Breast Reconstruction with Extended Latissimus Dorsi Muscle Flap
}

\author{
Kiyomi Tanaka Shoji Oura Koji Yasuda Shinichiro Makimoto \\ Department of Surgery, Kishiwada Tokushukai Hospital, Kishiwada, Japan
}

Keywords

Encapsulated seroma $\cdot$ Extended latissimus dorsi muscle flap $\cdot$ Minocycline

\begin{abstract}
A 57-year-old woman underwent salvage nipple-preserving mastectomy with immediate breast reconstruction using extended latissimus dorsi muscle flap for her in-breast recurrence. The patient had been well with a presumed encapsulated seroma in her back for 8 years and 3 months but suddenly developed a protrusion of the persistent seroma. The patient requested us to improve the cosmetic deterioration of the visible large protrusion. In the operation, the operative target was converted from the newly protruded portion to the whole persistent seroma due to the leakage of presumed contaminated fluid. The posterior wall of the long-lasting seroma sticked rigidly to the ribs, forcing us not to resect the whole capsule but to resect the anterior and lateral walls with scraping the posterior wall with a curet. Pathological study showed a dense fibrous capsule, amorphous eosinophilic material, cholesterin crystals, and massive histiocyte infiltration. Postoperative course was uneventful, but wound healing was not observed over 3 weeks after operation. Minocycline $100 \mathrm{mg}$ diluted in $20 \mathrm{~mL}$ saline was injected into the seroma cavity after full aspiration of the seroma fluid, causing immediate irritable sensation around the seroma cavity and complete disappearance of the seroma cavity in 3 weeks after the minocycline injection. Pathogenesis of this extremely rare complication remains uncertain, but long-lasting seroma formation should be avoided not to cause this type of late-phase complication. Minocycline injection into the seroma cavity is a feasible method to accelerate the wound healing.




\section{Introduction}

Breast cancer is one of the most common cancers worldwide. With the prevalence of screening mammography, breast cancer-related mortality has already dropped in some western countries [1]. Early detection of breast cancer not only improves the overall survival of the breast cancer patients, but can enable them to select their favorite therapy among various therapeutic options with similar curability.

With the advent of breast-conserving therapy $[2,3]$ and various newly developed anticancer agents, the therapeutic strategy of primary breast cancer has dramatically changed from unquestionable application of mastectomy to breast-conserving therapy, neoadjuvant chemotherapy followed by breast-conserving therapy, and primary or secondary, i.e. after neoadjuvant chemotherapy, (subcutaneous) mastectomy with breast reconstruction. Breast reconstruction consists of implant-based reconstruction and flap-based reconstruction. Each breast reconstruction has merits and demerits, leading to the appropriate application according to the patient's preference.

We report here an extremely rare case of abrupt aggravation of the encapsulated seroma occurring more than 8 years after salvage nipple-sparing mastectomy and immediate breast reconstruction using extended latissimus dorsi flap.

\section{Case Report}

A 57-year-old woman received salvage nipple-preserving mastectomy with immediate breast reconstruction using extended latissimus dorsi muscle flap for her breast recurrence in November 2011. Due to the prior exposure to anthracycline-containing chemotherapy and radiotherapy to the conserved breast, the patient received aromatase inhibitor after salvage surgery and showed favorable cosmetic outcome for a long time (Fig. 1a). Follow-up computed tomography (CT) showed no locoregional and distant metastases, but a presumed encapsulated seroma in her back for about 8 years (Fig. 2a). In February 2020, i.e. 8 years and 3 months after the salvage operation, the patient noticed a sudden elastic protrusion just under the skin incision on the back (Fig. 1b). CT showed a connection between the suddenly

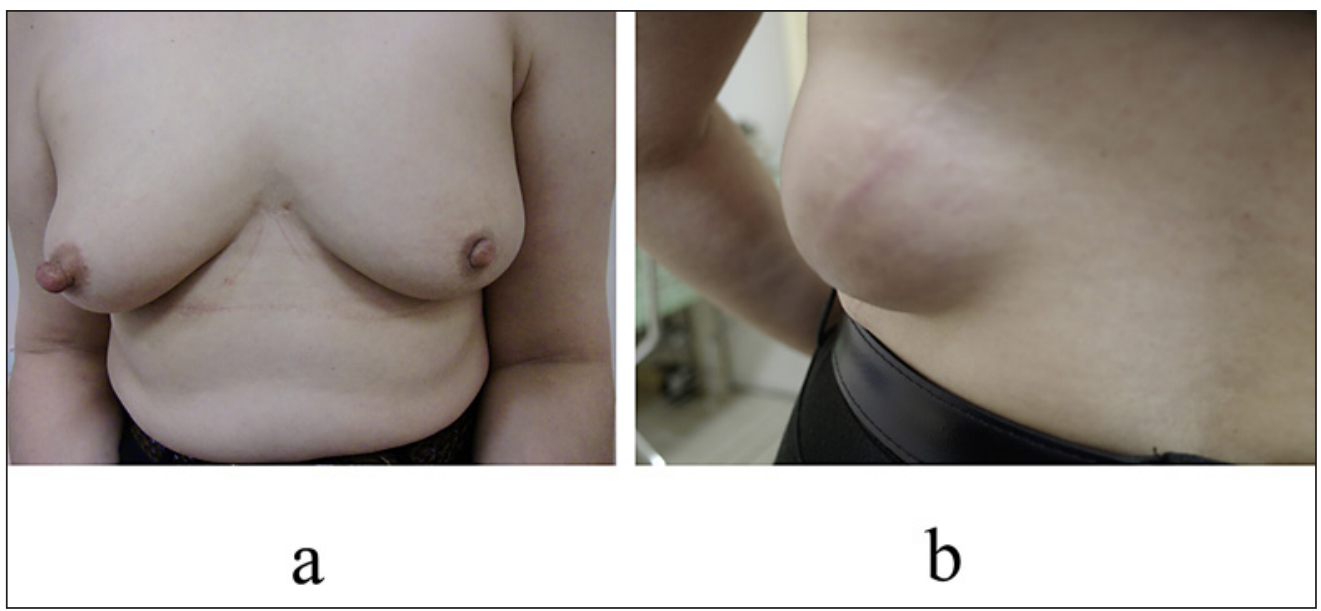

Fig. 1. Local findings. a Reconstructed left breast with extended latissimus dorsi muscle flap showed excellent cosmesis. b Marked skin elevation was observed just beneath the skin incision for harvesting the extended latissimus dorsi muscle flap. 


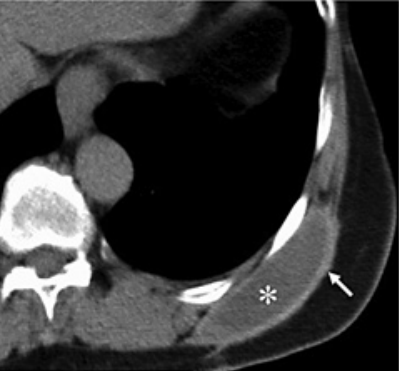

a

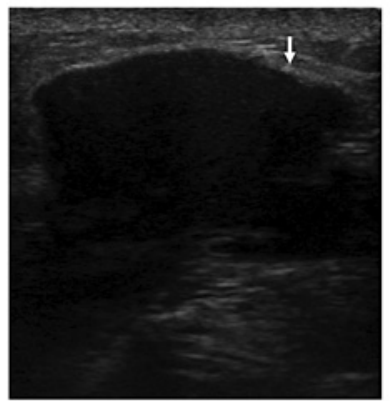

d

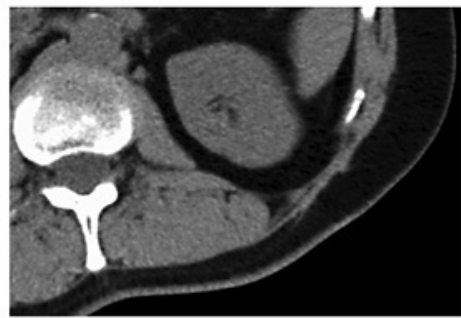

b

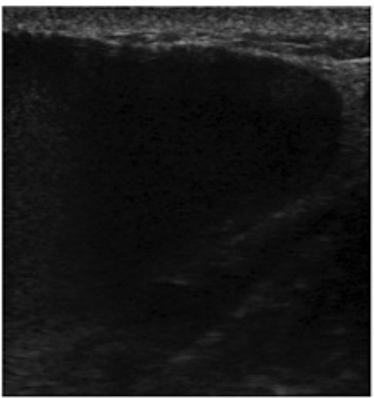

$\mathrm{e}$

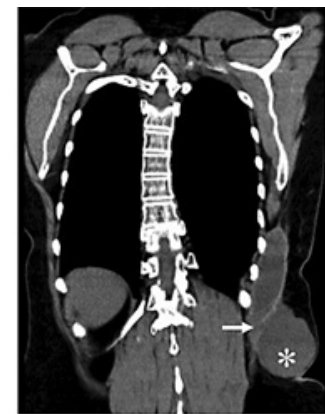

C

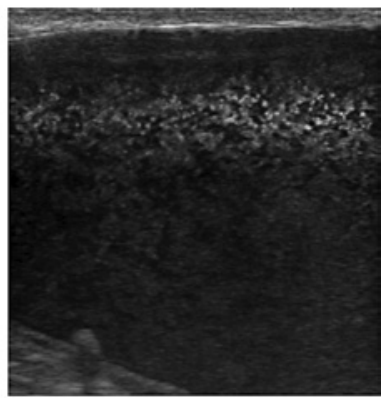

f

Fig. 2. Image findings around the seroma. a Computed tomography (CT) showed that the persistent seroma (asterisk) encompassed by thick capsule (arrow) was located just adjacent to the ribs. b CT showed that no apparent protrusion was observed up until 8 years after salvage operation. c Frontal view of the CT showed that the newly protruded presumed seroma (asterisk) was connected to the persistent seroma through the presumed rupture point (arrow). d Persistent seroma with thick capsule (arrow) was observed on ultrasound. e Apparent enlargement of seroma was observed on ultrasound. f Ultrasound showed numerous hyperechoic dots in the seroma.

protruded mass and the long-lasting seroma (Fig. 1c). Due to both the ultrasound findings (Fig. 2d,e) and the patient's request, we aspirated the fluid in the suddenly protruded portion of the seroma, leading to temporary disappearance of the protrusion. Aspirated liquid was serous and dark reddish-brown. Shortly after the liquid aspiration, elastic protrusion recurred in the back. Ultrasound showed that the internal echoes in the re-protruded old seroma had more hyperechoic dots (Fig. 2f) than those before the aspiration, suggesting the emersion of floating particles in the seroma. The patient requested us to improve the cosmetic deterioration and underwent surgery despite the unknown pathogenesis of the sudden protrusion of the persistent encapsulated seroma. We initially intended to resect only the newly protruded portion without the resection of long-lasting encapsulated seroma. Presumed contaminated fluid that leaked from the target portion made us change the resection target from the newly protruded portion to the whole seroma. However, it was impossible for us to safely resect the posterior wall of the long-lasting seroma sticking rigidly to the ribs, reluctantly forcing us to scrape the posterior wall with a curet. Pathological study showed a dense fibrous capsule, amorphous eosinophilic material, cholesterin crystals, and massive histiocyte infiltration (Fig. 3), strongly suggesting the presumed contaminated fluid not to be induced by bacterial infection. Postoperative course was uneventful, and the patient was discharged on the 10th day after the operation. After disharge, postoperative non-encapsulated seroma, however, 


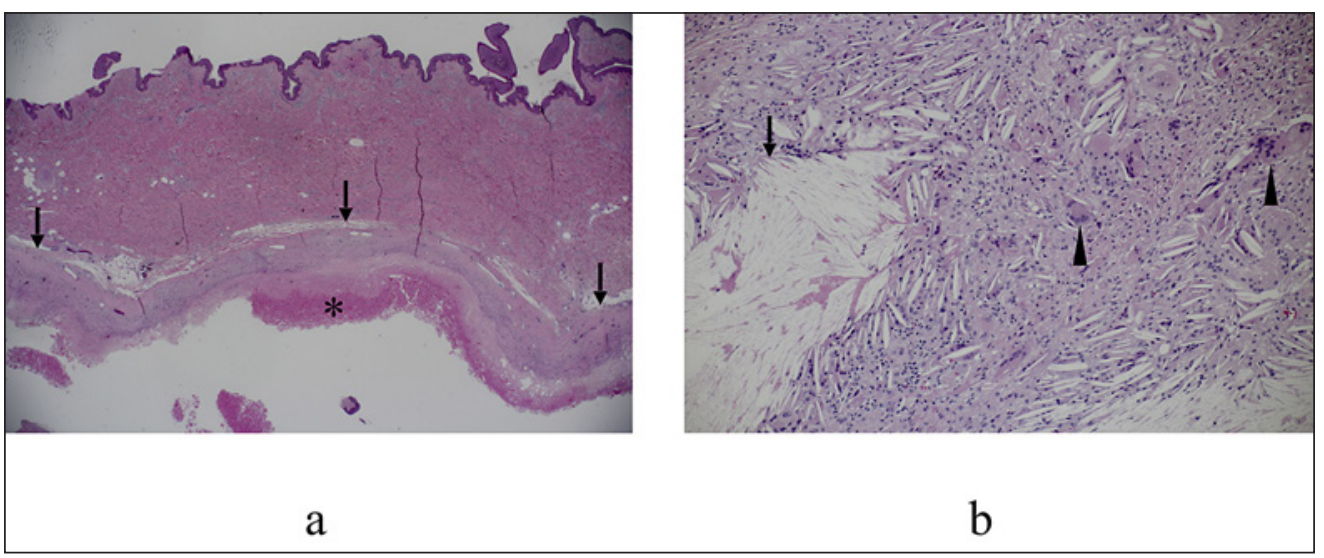

Fig. 3. Pathological findings. a Low-magnification view showed that the seroma was encompassed by thick hyalinized capsule (arrows) that contained amorphous eosinophilic material (asterisk). b The capsule contained massive cholesterol crystals (arrow) and foreign body multinucleate giant cells (arrowheads).

lasted for more than 3 weeks. Minocycline $100 \mathrm{mg}$ diluted in saline $20 \mathrm{~mL}$, therefore, was injected into the seroma cavity to accelerate the wound healing by chemical irritation after fully aspirating the retention fluid, causing immediate irritable feeling around the seroma cavity and complete disappearance of the seroma cavity in 3 weeks after the minocycline injection. The patient returned to the original follow-up scheme with aromatase inhibitor therapy.

\section{Discussion}

Implant-based breast reconstruction needs no additional skin incision and tissue harvesting [4]. Among various autologous tissues used for breast reconstruction, rectus abdominis muscle and latissimus dorsi muscle play major roles in the breast reconstruction. Rectus abdominis muscle, especially deep inferior epigastric artery perforator (DIEP) flap [5], can be easily moved to the target field and reconstruct a large breast. On the other hand, latissimus dorsi flap has stable blood flow, generally leading to safe breast reconstructions without flap loss [6].

In addition to the inevitable formation of capsular contracture, implant-based breast reconstruction, especially using textured type implant [7], has attracted both patients' and physicians' attention to the occurrence of breast implant-associated anaplastic large cell lymphoma [8]. Rectus abdominis muscle flap, especially DIEP flap, has unstable blood flow, sometimes leading to major or total flap loss. Latissimus dorsi flap under standard horizontal spindle skin incision on the back generally offers small amount of volume displacement. Latissimus dorsi muscle flap, therefore, is often used in the defect filling after breast-conserving surgery [9].

After nipple-preserving mastectomy through lateral vertical skin incision along with the breast contour, i.e. not visible from the front, extended latissimus dorsi flap was harvested from the back with an oblique spindle incision. Extended latissimus muscle flap, therefore, has a demerit of a visible skin incision on the back compared to the standard latissimus muscle flap reconstruction with invisible skin incision on the back when wearing a brassiere. On the other hand, extended latissimus dorsi muscle flap reconstruction, when harvested up to the

\section{Karger'}


lumbar fat around the iliac crest, dislocates relatively large volume of fat tissue with stable blood flow. In fact, cosmetic outcome in this case was excellent for more than 8 years after breast reconstruction. In addition, among more than 300 breast cancer patients reconstructed by us with extended latissimus dorsi flap, no patients developed total flap necrosis.

Displacement of latissimus dorsi muscle naturally creates a cavity between the skin and the thoraco-abdominal wall, inevitably forming seroma cavity until complete wound healing [10]. Prolonged seroma over several years itself is relatively rare but generally forms a capsule like implant-based breast reconstruction. Pathogenesis of the sudden aggravation of the persistent encapsulated seroma remains uncertain. Infection of the seroma due to the fluid aspiration could be a possible mechanism of this rare complication. Seroma aggravation, however, preceded the aspiration. In addition, laboratory data lacked inflammatory findings, and pathological evaluation showed some kind of inflammation in the capsule but at least no bacterial infection due to the lack of neutrophil infiltration.

Because smooth surfaces of the inner part of the encapsulated seroma do not adhere to each other, management of aggravated persistent seroma generally needs capsulectomy. Therefore, it is very important to avoid persistent seroma formation after the breast reconstruction. Possible measures to prevent the seroma formation include postoperative full drainage, appropriate aspiration of growing seroma followed by compression of the seroma cavity with some kind of girdle, and injection of some chemical irritants such as minocycline [11] and picibanil [12] into the seroma cavity.

In conclusion, extended latissimus dorsi muscle flap reconstruction is a feasible option for breast reconstruction, but much attention should be paid to the long-lasting seroma formation. Minocycline injection to the seroma cavity is a useful measure to accelerate the wound healing, leading to the prevention of persistent seroma formation.

\section{Statement of Ethics}

We have reported this case in compliance with the Declaration of Helsinki. Written informed consent was obtained from the patient for the publication of this case report and any accompanying images.

\section{Conflict of Interest Statement}

The authors have no conflicts of interest to declare.

\section{Funding Sources}

Not applicable.

\section{Author Contributions}

K.T. contributed the design of the report and collected the data. S.O. drafted the manuscript. K.Y. decided how to treat the patient. S.M. revised the manuscript. All authors have read and approved the final version of the manuscript. 


\section{References}

1 Habbema JD, van Oortmarssen GJ, van Putten DJ, Lubbe JT, van der Maas PJ. Age-specific reduction in breast cancer mortality by screening: an analysis of the results of the Health Insurance Plan of Greater New York study. J Natl Cancer Inst. 1986;77(2):317-20.

2 Veronesi U, Cascinelli N, Mariani L, Greco M, Saccozzi R, Luini A, et al. Twenty-year follow-up of a randomized study comparing breast-conserving surgery with radical mastectomy for early breast cancer. N Engl J Med. 2002;347:1227-32.

3 Fisher B, Anderson S, Bryant J, Margolese RG, Deutsch M, Fisher ER, et al. Twenty-year follow-up of a randomized trial comparing total mastectomy, lumpectomy, and lumpectomy plus irradiation for the treatment of invasive breast cancer. N Engl J Med. 2002;347(16):1233-41.

4 Eriksen C, Lindgren EN, Frisell J, Stark B. A prospective randomized study comparing two different expander approaches in implant-based breast reconstruction: one stage versus two stages. Plast Reconstr Surg. 2012; 130(2):254e-64e.

5 Granzow JW, Levine JL, Chiu ES, Allen RJ. Breast reconstruction using perforator flaps. J Surg Oncol. 2006; 94(6):441-54.

6 Menke H, Erkens M, Olbrisch RR. Evolving concepts in breast reconstruction with latissimus dorsi flaps: results and follow-up of 121 consecutive patients. Ann Plast Surg. 2001;47(2):107-14.

7 Araco A, Caruso R, Araco F, Overton J, Gravante G. Capsular contractures: a systematic review. Plast Reconstr Surg. 2009;124(6):1808-19.

8 McCarthy CM, Loyo-Berríos N, Qureshi AA, Mullen E, Gordillo G, Pusic AL, et al . Patient registry and outcomes for breast implants and anaplastic large cell lymphoma etiology and epidemiology (PROFILE): Initial report of findings, 2012-2018. Plast Reconstr Surg. 2019;143:1908-19.

9 Mele S, Wright D, Paramanathan N, Laws S, Peiris L, Rainsbury R. Long-term effect of oncoplastic breastconserving surgery using latissimus dorsi miniflaps on mammographic surveillance and the detection of local recurrence. J Plast Reconstr Aesthet Surg. 2017;70(9):1203-9.

10 Tomita K, Yano K, Masuoka T, Matsuda K, Takada A, Hosokawa K. Postoperative seroma formation in breast reconstruction with latissimus dorsi flaps: a retrospective study of 174 consecutive cases. Ann Plast Surg. 2007;59(2):149-51.

11 Lashevsky I, Ben Yosef R, Rinkevich D, Reisner S, Markiewicz W. Intrapericardial minocycline sclerosis for malignant pericardial effusion. Chest. 1996;109(6):1452-4.

12 Knipping S, Goetze G, Neumann K, Bloching M. Sclerotherapy of cervical cysts with Picibanil (OK-432). Eur Arch Otorhinolaryngol. 2007;264(4):423-7. 